\title{
Coplanar and grazing incidence $x$-ray-diffraction investigation of self-organized SiGe quantum dot multilayers
}

\author{
V. Holý,* A. A. Darhuber, J. Stangl, S. Zerlauth, F. Schäffler, and G. Bauer \\ Institute of Semiconductor Physics, Johannes Kepler University, Altenbergerstraße 69, A-4040 Linz, Austria \\ N. Darowski, D. Lübbert, ${ }^{\dagger}$ and U. Pietsch \\ Institute of Physics, University of Potsdam, Potsdam, Germany \\ I. Vávra \\ Institute of Electrical Engineering, Slovak Academy of Sciences, Dúbravská cesta 9, 84239 Bratislava, Slovakia
}

(Received 14 May 1998)

\begin{abstract}
We report on a formalism for the calculation of diffusely scattered x-ray intensity from spatially inhomogeneous strain fields in $\mathrm{Ge}$ rich islands and in the surrounding $\mathrm{Si}$ matrix of $\mathrm{SiGe} / \mathrm{Si}$ multilayers. The data analysis is based on a theory considering the two-dimensional statistical distribution of the dot positions, which allows a common formalism for both coplanar and grazing incidence scattering geometries. The strain fields were simulated based on the approach of the elastic Green function, taking the influence of the elastic strain relaxation at the sample surface into account. From these simulations the degree of relaxation of the islands was obtained, which compared very well with experimental data derived from x-ray reciprocal space maps. [S0163-1829(98)02736-2]
\end{abstract}

\section{INTRODUCTION}

Self-organized growth of nanoscale features driven by misfit strain ${ }^{1}$ in heteroepitaxial systems such as Ge/Si (Refs. 2-5) or InAs/GaAs (Refs. 6-8) has attracted much interest in recent years. It has been shown that misfit strain can cause the nucleation of quasi-zero-dimensional islands (quantum dots), ${ }^{9-11}$ of one-dimensional quantum wires, ${ }^{12}$ and of twodimensional periodic superlattice structures. ${ }^{13}$ In order to achieve a narrow size distribution of the nanostructures, which is a prerequisite for any possible technological application, several strategies have been reported. For Ge islands embedded in $\mathrm{Si}$ or for InAs in $\mathrm{GaAs},{ }^{7}$ it has been shown that multilayer structures provide an interesting route to achieve this goal. ${ }^{9}$ Buried dots influence through their strain fields the nucleation in subsequent layers, leading to vertical correlation of dot positions. Furthermore, through interaction of the strain fields, mediated by the Si layers, the $\mathrm{Ge}$ island separation and size in successive layers becomes progressively more uniform. ${ }^{9,13}$ The in-plane anisotropy of the elastic properties (Young's modulus) in diamond and zincblende structures favors a lateral ordering of the islands along two orthogonal [100] and [010] directions in the (001) growth plane. $8,10,14$

Previously, investigations on $\mathrm{Si} / \mathrm{SiGe}$ multilayers containing islands have been carried out by Baribeau, who used coplanar diffraction. ${ }^{15}$ Grazing incidence diffraction (GID), which is a powerful tool to get information on the depth dependent relaxation, ${ }^{16}$ has been employed by Williams et al. ${ }^{17}$ and Steinfort et al. ${ }^{18}$ for single dot Ge hut clusters and by Darhuber et al. for Si/SiGe multilayers. ${ }^{19}$ The shape transition of $\mathrm{Ge}$ nanocrystals on $\mathrm{Si}(001)$ from pyramides to domes has been investigated by Medeiros-Ribeiro et al. ${ }^{20}$ Recently, Kegel et al. have used GID and small-angle scattering techniques to obtain information both on the shape and on the strain status of uncovered InAs dots on GaAs. ${ }^{21}$ It is by now well established that the strain fields act as driving forces for the vertical as well as the lateral correlations of islands in multilayers. For the determination of such strain fields x-ray-diffraction techniques are most suitable, which have been mainly applied so far for the investigation of etched nanostructures. ${ }^{22}$

In this paper we present a theory for the analysis of the diffuse scattering from self-organized dots embedded in a matrix. So far only a one-dimensional statistical distribution of dots has been considered, which is consequently not adequate for the simulation of grazing incidence diffraction. In order to calculate the diffusely scattered intensity both for the coplanar [high-resolution x-ray diffraction (HRXRD)] as well as for the grazing incidence (GID) scattering geometry, a two-dimensional statistical distribution of the Ge-island positions was considered, thus enabling calculations for both scattering geometries. The elastic distortions both within the Ge rich islands and in the surrounding Si matrix are considered. Measured reciprocal space maps were compared with calculated ones based on statistical kinematic scattering theory and distorted wave Born approximation (DWBA). ${ }^{23,24}$ Thus we present a unified description of coplanar and grazing incidence diffraction experiments, in which a single set of position-dependent parameters derived from elasticity theory describes both the HRXRD and the GID data set. The inhomogeneous deformation fields of the dots embedded in the Si matrix were calculated based on the approach of the elastic Green function. This theory has been applied to the analysis of diffraction data obtained on a series of molecular beam epitaxy (MBE) grown ten-period $\mathrm{Si} / \mathrm{SiGe}$ multilayers. Cross-sectional transmission electron micrography (TEM) has been employed to determine the dot sizes. Information on the strain fields present within the Ge rich islands as well as in the surrounding Si matrix was obtained from coplanar 
TABLE I. Nominal structural parameters of the investigated samples and the lateral period of the dot arrays determined from the RSM's.

\begin{tabular}{lccccr}
\hline \hline Sample & $T\left({ }^{\circ} \mathrm{C}\right)$ & $x_{\mathrm{Ge}}$ & $d_{\mathrm{SiGe}}(\mathrm{nm})$ & $d_{\mathrm{Si}}(\mathrm{nm})$ & $L(\mathrm{~nm})$ \\
\hline SiGe44 & 700 & 0.48 & 2.0 & 20.0 & $190 \pm 10$ \\
SiGe51 & 600 & 0.51 & 2.1 & 20.0 & $110 \pm 10$ \\
SiGe53 & 600 & 0.40 & 2.0 & 20.5 & $110 \pm 10$ \\
SiGe63 & 500 & 0.61 & 2.3 & 14.7 & $80 \pm 10$ \\
\hline \hline
\end{tabular}

and GID experiments. By recording reciprocal space maps around several reciprocal lattice points with the latter method the three-dimensional scattered intensity distribution could be monitored.

The paper is organized as follows. In Sec. II the $\mathrm{Si} / \mathrm{SiGe}$ multilayer dot samples are described and experimental data from TEM, HRXRD, and GID are presented. In Sec. III the calculation of the deformation field for the island multilayer samples is described as well as the calculation of the intensities of the diffusely scattered radiation from the inhomogeneously strained system. In Sec. IV we outline in particular the comparison between the calculations and the experimental data. The simulations based on elasticity theory yield the degree of relaxation of the three-dimensional dot lattice, which compares very well with the experimental data derived from the reciprocal space maps both from HRXRD and from GID.

\section{EXPERIMENT}

\section{A. Sample preparation}

Several ten-period $\mathrm{Si}_{1-x} \mathrm{Ge}_{x} / \mathrm{Si}$ multilayers were grown by MBE at growth temperatures between $T=500{ }^{\circ} \mathrm{C}$ and $700{ }^{\circ} \mathrm{C}$ onto $\mathrm{Si}(001)$. The germanium content of the nominally $2.0-2.3 \mathrm{~nm}$ thick $\mathrm{SiGe}$ layers varied between $x_{\mathrm{Ge}}$ $=0.4$ and 0.6. The thickness of the Si layers ranged from 15 to $20 \mathrm{~nm}$ and the Si capping layer thickness was about 20 $\mathrm{nm}$. The nominal parameters of the samples are summarized in Table I.

Figure 1 shows a cross-sectional transmission electron micrograph of sample SiGe51. As shown, the dots are correlated in the lateral and vertical directions. The average dot height is about $4-5 \mathrm{~nm}$. The determination of the average distance of the dots is complicated by the small size of the depicted volume and by the fact that we can only see the projections of the dot positions. However, we can roughly estimate the mean lateral dot distance to be $150 \pm 50 \mathrm{~nm}$.

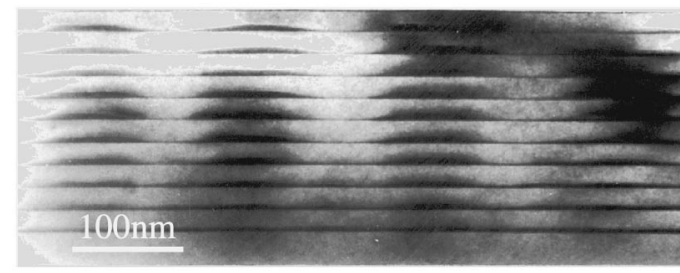

FIG. 1. Cross-sectional transmission electron micrograph of sample $\mathrm{SiGe51.} \mathrm{The} \mathrm{dark} \mathrm{lines} \mathrm{and} \mathrm{islands} \mathrm{are} \mathrm{the} \mathrm{SiGe}$ regions. The SiGe wetting layers and the vertically correlated islands are clearly visible.

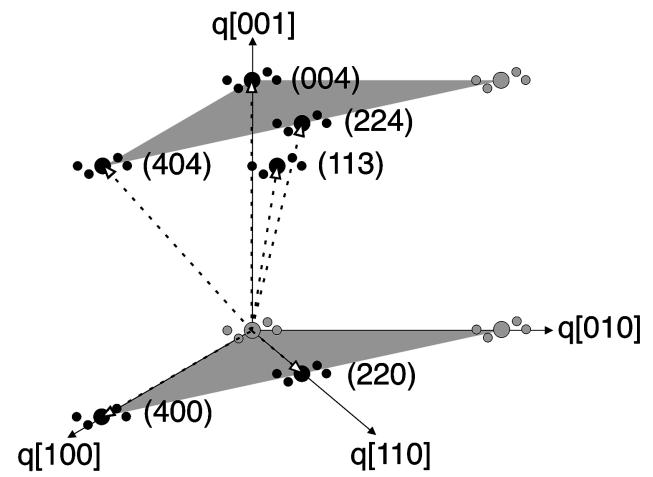

FIG. 2. Sketch of the reciprocal lattice of the dot samples. The larger dots show the fundamental Bragg reflections that are accessible by coplanar and grazing incidence diffraction. The smaller dots depict the satellites due to the lateral ordering within the (001) growth plane. The satellites due to the vertical periodicity have been omitted for clarity.

\section{B. Coplanar diffraction}

HRXRD measurements have been performed with a Philips materials research diffractometer equipped with a fourcrystal $\mathrm{Ge}(220)$ monochromator and a channel-cut $\mathrm{Ge}(220)$ analyzer. The wavelength was $\lambda=0.15406 \mathrm{~nm}$ with a resolution of $\Delta \lambda / \lambda=1 \times 10^{-4}$. The angular resolution of this setup is 12 arcsec. Reciprocal space maps (RSM's) around various symmetric and asymmetric reciprocal lattice points [(004), (113), (224), and (404)] were recorded. Figure 2 shows a sketch of the reciprocal lattice with the reciprocal lattice points, which have been measured in coplanar and in grazing incidence diffraction. Depicted are the fundamental Bragg reflections together with first-order satellites due to the lateral ordering along two orthogonal $\langle 100\rangle$ directions. The satellites due to the periodicity along growth direction are omitted for the sake of clarity.

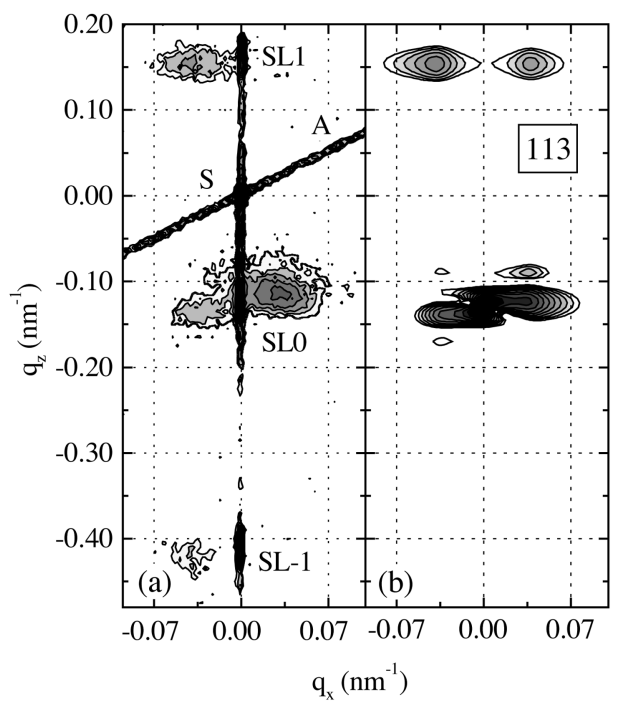

FIG. 3. (a) Measured and (b) simulated RSM in coplanar HRXRD geometry, sample SiGe51, and symmetrical diffraction (113). $\mathrm{SL}_{-1}, \mathrm{SL}_{0}$, and $\mathrm{SL}_{1}$ denote the negative first, zeroth, and positive first superlattice satellites, respectively, $S$ is the substrate peak, and $A$ denotes the analyzer streak. The intensity contours are plotted in steps of $\Delta I=10^{0.25}$. The calculated RSM shows only the diffusely scattered intensity distribution. 


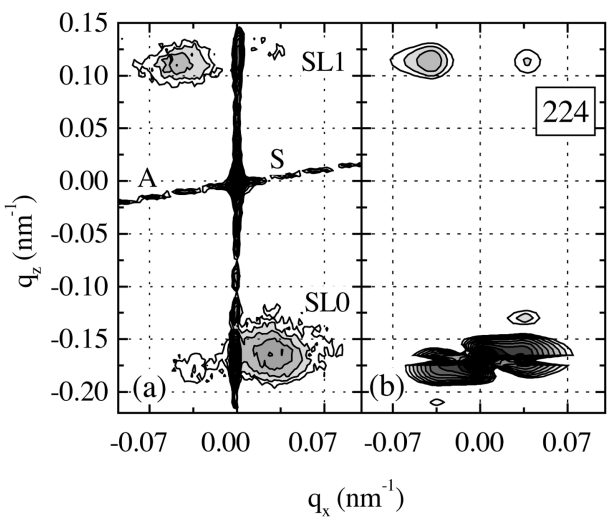

FIG. 4. Same as Fig. 3, but for asymmetrical diffraction (224).

Figures 3(a)-5(a) show RSM's of the asymmetrical Bragg reflections for a sample SiGe51 (with $x_{\mathrm{Ge}}=0.5$ grown at $T=600{ }^{\circ} \mathrm{C}$ ). The oblique line is the so-called analyzer streak and does not contain structural information. Along $q_{z}$ all the recorded RSM's display pronounced zeroth and positive first satellite peaks. In the (113) RSM also the negative first-order satellite peak is visible (Fig. 3). The satellite peaks reflect the vertical correlation of the electron density. The multilayer period is determined to be $D=2 \pi / \Delta q_{z}=22.4$ $\pm 0.5 \mathrm{~nm}$. Due to the vertical strain of the average multilayer with respect to the silicon substrate the whole pattern of satellite peaks is shifted by $\Delta q_{z} \approx-0.16 \mathrm{~nm}^{-1}$ (in 224 and 404) and by $-0.12 \mathrm{~nm}^{-1}$ (in 113) from the silicon reciprocal lattice point. This shift is proportional to the vertical component $h_{z}$ of the diffraction vector.

Distinct side peaks appear in the asymmetrical RSM's, which are related to the in-plane correlation of dots. Due to the lateral lattice strain acting at the dots, their intensity distribution is asymmetric with respect to $q_{x}=0$. Whereas the zeroth-order satellite shows mainly compressive strain (the main maximum is displaced to larger $q_{x}$ ), the first-order peaks appear rather expanded because the main maximum appear at smaller $q_{x}$. In Figs. 6(a) and 6(b) we have plotted the distribution of the diffusely scattered intensity along the horizontal lines $q_{z}=$ const in reciprocal space crossing the zeroth [Fig. 6(a)] and the positive first multilayer satellite [Fig. 6(b)]. In order to suppress the noise, the intensity has been integrated in the $q_{z}$ direction in the range $\Delta q_{z}$ $=0.05 \mathrm{~nm}^{-1}$. Figure 7 shows the similar intensity distribu-

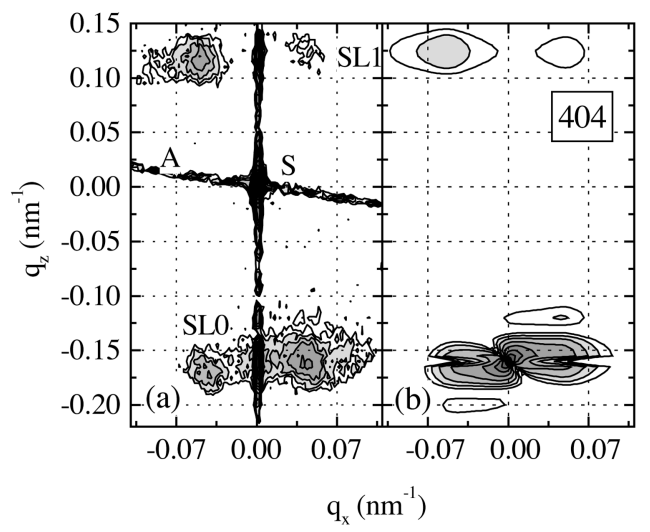

FIG. 5. Same as Fig. 3, but for asymmetrical diffraction (404).

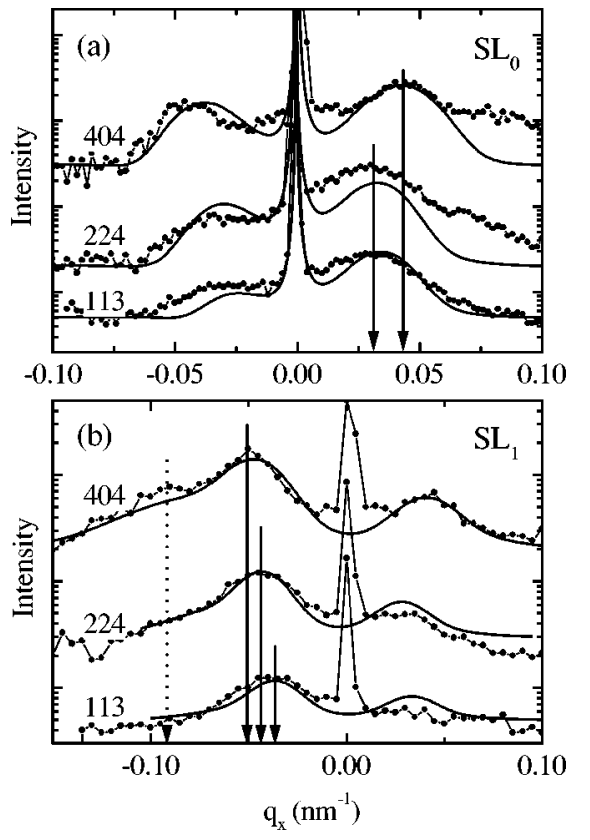

FIG. 6. Measured (points) and calculated (full lines) intensity distributions along horizontal lines crossing the (a) zeroth and (b) positive first lateral satellites. The measured intensities have been integrated in the range $\Delta q_{z}=0.05 \mathrm{~nm}^{-1}$ in order to reduce the noise. Sample SiGe51, diffractions 113, 224, and 404. The curves of 224 and 404 are shifted upward. The vertical lines denote the positions of the lateral satellites of the first (full) and second (dotted) orders.

tions measured from various samples close to the zeroth satellite [Fig. 7(a)] and the positive first satellite [Fig. 7(b)]. As shown in Fig. 7(a), the lateral spacing of the dots increases with increasing growth temperature and decreases with increasing Ge concentration. In Fig. 7(b) the separation be-

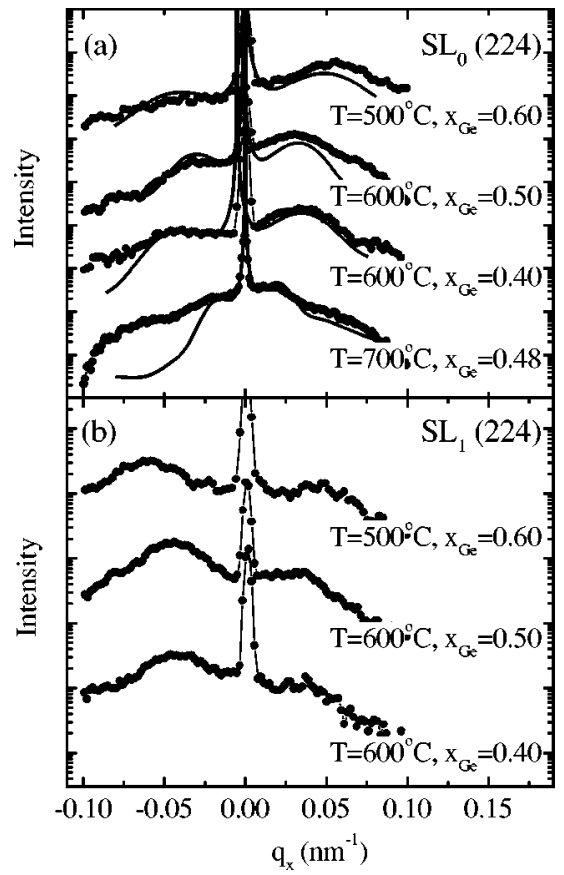

FIG. 7. Similar to Fig. 6, but for experimental curves of samples SiGe44, SiGe51, SiGe53, and SiGe63. 


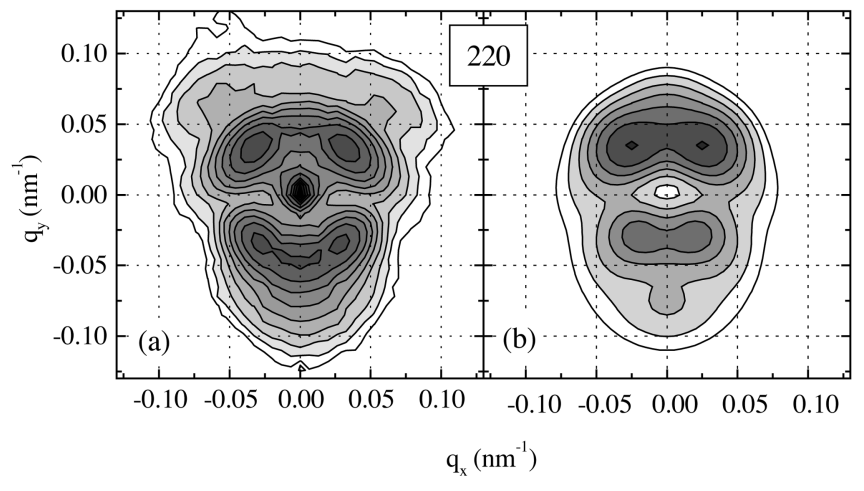

FIG. 8. In-plane $q_{x} q_{y}$ RSM of sample SiGe51 around (220) Bragg reflection in GID geometry: (a) measurement and (b) simulation. The diffraction vector is parallel to the positive $q_{y}$ axis. The step in the intensity contours is $\Delta I=10^{0.25}$.

tween the narrow peak of the coherent truncation rod and the broad dot peak increases for increasing Ge concentration.

\section{Grazing incidence diffraction}

The GID experiments were performed at the wiggler beam line BW2 at HASYLAB, Hamburg. The wavelength of the parallel and monochromatic incident beam was $\lambda$ $=0.13627 \mathrm{~nm}$. The resolution was increased by using a $\mathrm{Si}(111)$ analyzer crystal. The angular resolutions of the angle of incidence $\alpha_{i}$ and exit $\alpha_{f}$ were better than $0.001^{\circ}$. Because of this high resolution we were able to measure reciprocal space maps corresponding to features in different depths below the sample surface. ${ }^{16,25}$ The coordinates of the reciprocal space are oriented as follows: $q_{x}$ and $q_{y}$ axes are aligned along the [110] and [1 $1 \overline{1} 0]$ directions of the substrate and $q_{z}$ is parallel to the surface normal. The penetration depth is controlled by $\alpha_{i}$, which can be chosen to be smaller or larger than the critical angle of total external reflection $\alpha_{c}$. For $\alpha_{i}<\alpha_{c}$ the x-ray wave is evanescent and propagates parallel to the surface with a penetration depth of about $5 \mathrm{~nm}$. Increasing $\alpha_{i}$ above $\alpha_{c}$, the x-ray wave can propagate through the sample, i.e., the penetration depth can exceed several hundreds of nanometers.

Maps around the (220) and (400) reciprocal lattice points were recorded (see Fig. 2). In order to enhance the sensitivity to the strain fields in the surface-near region, we measured mainly under evanescent condition $\alpha_{i}<\alpha_{c}$. Figure 8 shows the (220) RSM recorded in the $q_{x^{-}} q_{y}$ plane for $\alpha_{i} \approx \alpha_{c}$ corresponding to a penetration depth of about $15-20 \mathrm{~nm}$. Thus the upper SiGe layer and the silicon capping layer are probed only. The RSM shows clearly the dot correlation direction along $\langle 100\rangle$. The map appears symmetric with respect to the $q_{y}$ axis, which corresponds to the radial scan direction (changing length of the scattering vector). Along $q_{y}$ the intensity distribution appears slightly asymmetric caused by the influence of in-plane strain. Its depth variation is shown in Fig. 9, displaying the RSM within the $q_{y}-q_{z}$ plane. Beside the crystal truncation rod of the silicon substrate at $q_{y}=0$, the $q_{z}$ variation of the self-organized dots are clearly visible, displayed as side peaks with respect to the truncation rod of the substrate. Whereas the side peaks corresponding to tensile strain remain, the compressed counterpart disappears with increasing $q_{z}$.

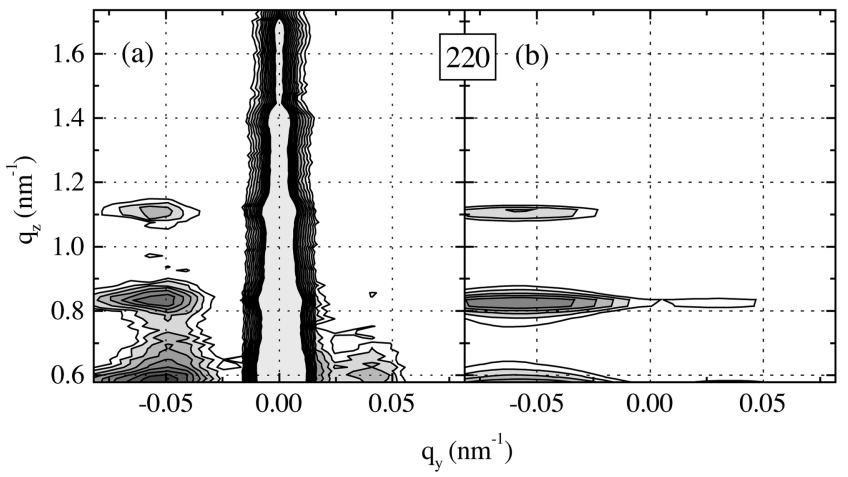

FIG. 9. Vertical $q_{y} q_{z}$ RSM around the (220) reciprocal lattice point in GID geometry.

Finally Fig. 10 shows the in-plane RSM recorded within the $q_{x}-q_{y}$ plane close to the ( $\left.\overline{4} 00\right)$ diffraction. The penetration depth is restricted again to the topmost layers. The $q_{y}$ axis is again parallel to [110]. In this figure, the radial [100] direction runs from the lower right to the upper left corner, i.e., rotated by $-45^{\circ}$ with respect to $q_{y}$. The lateral satellites caused by the ordering of the dot positions appear only along the radial direction, whereas, unlike (220) diffraction, they disappear completely in the angular direction. This difference between (220) and (400) RSM's is explained in Sec. IV.

Due to the chosen geometrical conditions the GID RSM's (Figs. 8 and 10) contain information about the correlation and strain in the topmost layer since they have been measured with an angle of incidence $\alpha_{i}=\alpha_{c}$. Separate scans along the truncation rods as functions of $\alpha_{i}$ (Fig. 11) demonstrate that multilayer satellites appear only for $\alpha_{i}$ larger than $\alpha_{c}$.

\section{THEORETICAL DESCRIPTION}

In this section we describe the calculation of the deformation of a dot embedded in a semi-infinite matrix and the deformation field caused by the dot in its neighborhood. Then we introduce the two-dimensional correlation function of the dot positions and finally we present a formula for the reciprocal space distribution of the diffusely scattered intensity. We took a two-dimensional statistical distribution of the

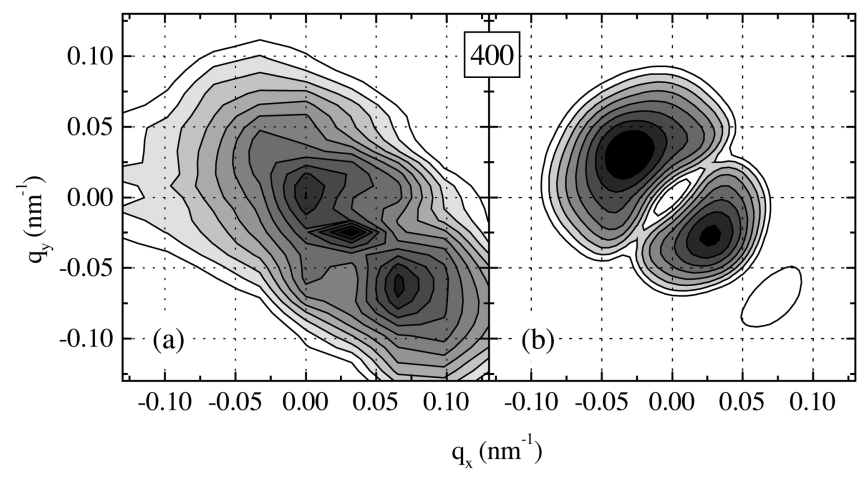

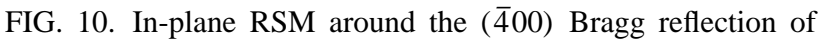
sample SiGe51 in GID geometry: (a) measurement and (b) simulation. The diffraction vector points from the lower right to the upper left corner of the picture $\left(q_{x}\left\|[110], q_{y}\right\|[\overline{1} 10]\right)$. 


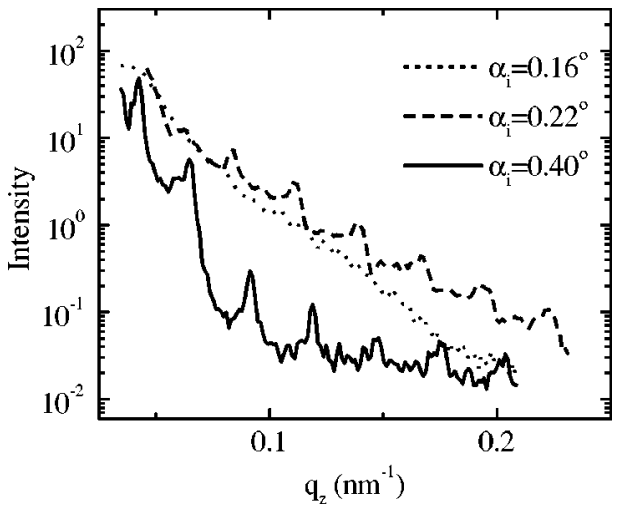

FIG. 11. Dependence of the scattered intensity on $q_{z}(\|[001])$ in GID geometry (scans along the truncation rod) measured in diffraction (220) for various incidence angles $\alpha_{i}$ (sample SiGe51).

dot positions into account. This allowed us to use a common formalism for both the coplanar and GID scattering geometries.

\section{A. Calculation of the deformation field}

The calculation of the deformation field is based on the approach of the elastic Green function. ${ }^{26}$ This method neglects the elastic anisotropy as well as the differences in the elastic constants of the dot and the surrounding matrix and it assumes that the semi-infinite sample has a perfectly flat surface.

Within this approach, the displacement $\mathbf{u}(\mathbf{r})$ is calculated as a superposition of the contributions of "point sources" of deformation

$$
\mathbf{u}(\mathbf{r})=\int d^{3} \mathbf{r}^{\prime} \Omega\left(\mathbf{r}^{\prime}\right) \mathbf{g}\left(\mathbf{r}, \mathbf{r}^{\prime}\right),
$$

where $\Omega(\mathbf{r})$ is the shape function of the volume containing the point sources (i.e., unity in the SiGe volume of the sample and zero in $\mathrm{Si}$ ) and

$$
\begin{aligned}
\mathbf{g}\left(\mathbf{r}, \mathbf{r}^{\prime}\right)= & \frac{P}{4 \pi}\left[\frac{\mathbf{R}_{1}}{R_{1}^{3}}-(3-4 \nu) \frac{\mathbf{R}_{2}}{R_{2}^{3}}-6 z\left(z+z^{\prime}\right) \frac{\mathbf{R}_{2}}{R_{2}^{5}}\right. \\
& \left.-2\left[(3-4 \nu)\left(z+z^{\prime}\right)-z\right] \frac{\mathbf{n}}{R_{2}^{3}}\right]
\end{aligned}
$$

is the elastic Green function expressing the contribution of the point source (point defect) in point $\mathbf{r}^{\prime}$ to the shift $\mathbf{u}(\mathbf{r})$. We have denoted

$$
\mathbf{R}_{1}=\left(x-x^{\prime}, y-y^{\prime}, z-z^{\prime}\right), \quad \mathbf{R}_{2}=\left(x-x^{\prime}, y-y^{\prime}, z+z^{\prime}\right),
$$

and

$$
P=x_{\mathrm{Ge}} \delta \frac{1+\nu}{1-\nu}
$$

$\delta$ is the lattice mismatch between Ge and the Si crystal matrix, $\nu$ is the Poisson ratio, $x_{\mathrm{Ge}}$ is the Ge concentration in the $\mathrm{SiGe}$ dots, the $z$ axis is parallel to the inward surface normal, and $\mathbf{n}$ is the unit vector parallel to this axis.

Equations (1) and (2) allow us to calculate the deformation field of an arbitrary shape $\Omega(\mathbf{r})$ of the SiGe regions.

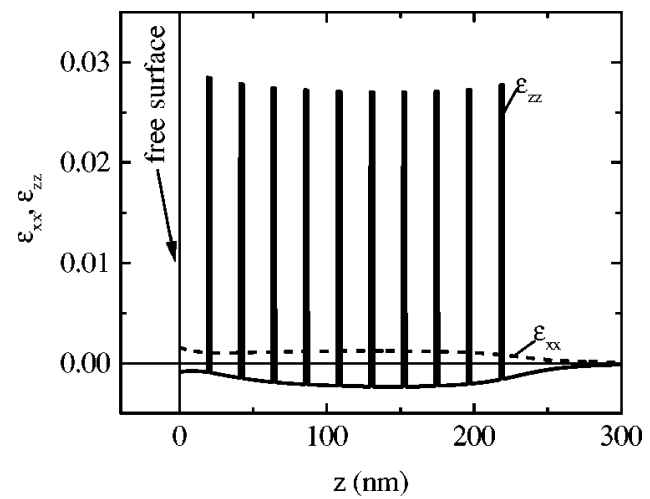

FIG. 12. Dependence of the strains $\varepsilon_{x x, z z}$ on the depth $z$ below the sample surface along the common axis of the dots. A single column of cylindrical dots with dot radius $R=40 \mathrm{~nm}$, dot height $h=2 \mathrm{~nm}$, and Ge content $x_{\mathrm{Ge}}=45 \%$. The regions with increased $\varepsilon_{z z}$ correspond to the dot volumes.

However, a direct analytical calculation is possible only for the simplest cases. Therefore, we approximated the dot shape by a cylinder with the radius $R$ and height $h$ and we calculated the deformation field of a vertical row of dots lying in the depths $Z_{n}=T_{c}+(n-1) D, n=1, \ldots, N$, where $T_{c}$ is the thickness of the capping layer at the sample surface and $D$ is the superlattice period. This calculation can be performed analytically only for a single vertical column of dots and along the common rotational $z$ axis of the dots. Numerical estimates have shown that the influence of dots in other vertical columns on the strain distribution in the particular dot column can be neglected. The wetting layers on the bottom of the dots play nearly no role and they have been completely ignored.

Figure 12 shows the dependences of the components $\varepsilon_{x x}$ and $\varepsilon_{z z}$ of the strain tensor $\hat{\varepsilon}$ on $z$ along the dot axis calculated for $N=10, T_{c}=20 \mathrm{~nm}, D=22.1 \mathrm{~nm}, R=40 \mathrm{~nm}, h$ $=2 \mathrm{~nm}$, and $x_{\mathrm{Ge}}=45 \%$. In the following, these values have been used for the simulation of the scattered intensity. The strain elements are defined with respect to the Si crystal matrix; therefore, $\varepsilon_{z z}$ equals nearly the lattice mismatch in the $\mathrm{SiGe}$ dots. Since the structure is assumed to be pseudomorphic, the value of $\varepsilon_{x x}$ averaged over the SiGe-Si interface must be zero. Therefore, the positive values of $\varepsilon_{x x}$ along the dot axis indicate the presence of stressed $\mathrm{Si}$ regions $\left(\varepsilon_{x x}\right.$ $<0$ ) outside the column of the dots. From Fig. 12 the influence of the surface stress relaxation is clearly visible. Its influence is significant only for the two upper dot layers of our samples.

From these calculations, the strains in the dots can be determined. Unfortunately, it is nearly impossible to use Eq. (1) for the calculation of the displacement distribution in a general point outside the rotation axis of the dot. For this purpose we have used a method published previously in Ref. 27 , where an expression was derived for the Fourier transformation of the function $\phi_{0}\left(\mathbf{r}, \mathbf{r}^{\prime}\right)=\mathbf{h} \cdot \mathbf{g}\left(\mathbf{r}, \mathbf{r}^{\prime}\right)$. $\mathbf{h}$ denotes the diffraction vector and the surface relaxation is taken into account. From the translational symmetry of the problem $\phi_{0}\left(\mathbf{r}, \mathbf{r}^{\prime}\right)=\phi_{0}\left(\mathbf{r}-\mathbf{r}^{\prime} ; z^{\prime}\right)$ follows. The Fourier transform of this function is given by 


$$
\begin{aligned}
\phi_{0}^{\mathrm{FT}}\left(\mathbf{q} ; z^{\prime}\right) & =\int d^{3} \mathbf{r} \phi_{0}\left(\mathbf{r} ; z^{\prime}\right) e^{i \mathbf{q} \cdot \mathbf{r}} \\
& =i P\left[\frac{\mathbf{h} \cdot \mathbf{q}}{q^{2}}-\Phi(\mathbf{q}) e^{-\left(q_{\|}+i q_{z}\right) z^{\prime}}\right],
\end{aligned}
$$

where the first term on the right-hand side represents the Fourier transformation of the deformation field of a point defect in an infinite matrix and the second term accounts for the surface strain relaxation. The function $\Phi$ is given by

$$
\begin{aligned}
2 \Phi(\mathbf{q})= & \left(\frac{1}{q_{\|}+i q_{z}}-\frac{3-4 \nu}{q_{\|}-i q_{z}}\right)\left(\frac{\mathbf{h}_{\|} \cdot \mathbf{q}_{\|}}{\left|\mathbf{q}_{\|}\right|}+i h_{z}\right) \\
& +\frac{2 q_{\|}}{\left(q_{\|}-i q_{z}\right)^{2}}\left(\frac{\mathbf{h}_{\|} \cdot \mathbf{q}_{\|}}{\left|\mathbf{q}_{\|}\right|}-i h_{z}\right) .
\end{aligned}
$$

Let us denote $\phi(\mathbf{r}, \mathbf{R})=\mathbf{h} \cdot \mathbf{u}(\mathbf{r}, \mathbf{R})$, where $\mathbf{u}(\mathbf{r}, \mathbf{R})$ is the displacement at point $\mathbf{r}$ due to the dot centered at point $\mathbf{R}$ $=(X, Y, Z)$. Then, using Eq. (1) and assuming that the dot volume is so small that the influence of surface relaxation is the same at any point inside the dot, we obtain ${ }^{14}$

$$
\phi^{\mathrm{FT}}(\mathbf{q} ; Z)=\int d^{3} \mathbf{r} \phi(x, y, z, Z) e^{i \mathbf{q} \cdot \mathbf{r}} \approx \Omega_{D}^{\mathrm{FT}}(\mathbf{q}) \phi_{0}^{\mathrm{FT}}(\mathbf{q} ; Z),
$$

where $\Omega_{D}(\mathbf{r})$ is the shape function of a single dot and $\Omega^{\mathrm{FT}}(\mathbf{q})$ is its Fourier transform. Equation (5) will be used for the following calculation of the diffusely scattered intensity.

\section{B. Intensity of the scattered wave}

In this section we derive an expression that describes the distribution of the diffusely scattered intensity $I(\mathbf{q})$ in reciprocal space. The position vector $\mathbf{q}$ is determined by the wave vectors of the primary and scattered waves and by the diffraction vector $\mathbf{h}$,

$$
\mathbf{q}=\mathbf{K}_{\mathrm{out}}-\mathbf{K}_{\mathrm{in}}-\mathbf{h}
$$

In the following we will not deal with the wave diffracted from a homogeneous, perfect sample. This wave is distributed along the line $q_{x}=0$ in reciprocal space (truncation rod) and it has to be calculated by means of dynamical diffraction theory. This was the reason why we did not take the wetting layers into account in the previous sections. The waves with $q_{x} \neq 0$ contribute to the diffusely scattered wave; these waves can be calculated using the kinematic diffraction theory (coplanar HRXRD) or by the DWBA approach (for GID geometry).

If the sample contains a single dot in position $\mathbf{R}$, the amplitude of the diffusely scattered wave will be distributed in reciprocal space according to

$$
E_{1}(\mathbf{q} ; \mathbf{R})=e^{i \mathbf{q} \cdot \mathbf{R}} \int d^{3} \mathbf{r} \chi_{h}(\mathbf{r}) e^{i \phi(\mathbf{r} ; Z)} e^{i \mathbf{q} \cdot \mathbf{r}} \equiv e^{i \mathbf{q} \cdot \mathbf{R}} E_{0}(\mathbf{q} ; Z),
$$

where $\chi_{h}(\mathbf{r})$ is the polarizability coefficient of the diffraction $\mathbf{h}$; we assume that $\chi_{h}(\mathbf{r})=\chi_{D}$ in the dot and $\chi_{L}$ in the surrounding crystal. Inside the dot we neglect the inhomogeneities in the strain distribution so that the shifts $\mathbf{u}(\mathbf{r})$ are linear functions of position. Outside the dot we will assume that the deformation field is weak so that $\exp [i \mathbf{h} \cdot \mathbf{u}(\mathbf{r})] \approx 1+i \mathbf{h}$ $\cdot \mathbf{u}(\mathbf{r})$. Then

$$
E_{0}(\mathbf{q} ; Z)=i \phi_{0}^{\mathrm{FT}}(\mathbf{q} ; Z) \Omega_{D}^{\mathrm{FT}}(\mathbf{q}) \chi_{L}+\left(\chi_{D}-\chi_{L}\right) \Omega_{D}^{\mathrm{FT}}\left[\mathbf{q}^{\prime}(Z)\right],
$$

where $q_{j}^{\prime}=q_{j}+h_{i} \varepsilon_{i j}(Z)$ is the reciprocal space vector corrected by the strain inside the dot. This strain was calculated by means of Eqs. (1) and (2) and it depends on the depth $Z$ of the dot below the surface (see Fig. 12).

If the sample contains a large number of dots, the total diffusely scattered intensity is averaged over all dot positions:

$$
I(\mathbf{q})=\left\langle\sum_{\mathbf{R}} \sum_{\mathbf{R}^{\prime}} E_{0}(\mathbf{q} ; Z) E_{0}^{*}\left(\mathbf{q} ; Z^{\prime}\right) e^{i \mathbf{q} \cdot\left(\mathbf{R}-\mathbf{R}^{\prime}\right)}\right\rangle .
$$

In order to calculate this average, a description of the statistical distribution of the dot positions is required. At a given interface, the distribution of the dots is not fully regular and it can be described by the Fourier transformation $w^{\mathrm{FT}}\left(\mathbf{q}_{\|}\right)$of the probability density $w\left(\mathbf{R}_{\|}\right)$:

$$
w^{\mathrm{FT}}\left(\mathbf{q}_{\|}\right)=\left\langle\sum_{\mathbf{R}_{\|}} e^{i \mathbf{q}_{\|} \cdot \mathbf{R}_{\|}}\right\rangle .
$$

If the dots created a perfect square array, this function would equal a series of $\delta$ functions $w^{\mathrm{FT}}\left(\mathbf{q}_{\|}\right) \sim \sum_{\mathbf{m}} \delta\left(\mathbf{q}_{\|}-m_{1} \mathbf{G}_{1}\right.$ $-m_{2} \mathbf{G}_{2}$ ), where $\mathbf{G}_{1,2}$ are the unit vectors of the reciprocal array. Let us assume now that these unit vectors are random, their mean values are $\left\langle\mathbf{G}_{1}\right\rangle=(G, 0,0)$ and $\left\langle\mathbf{G}_{2}\right\rangle=(0, G, 0)$, where $G=2 \pi / L$, and $L$ is the mean distance of the dots. If we assume that the probability distribution functions $P_{\sigma}\left(\mathbf{G}_{1,2}\right)$ correspond to the two-dimensional normal distributions with the dispersion $\sigma^{2}$, we obtain

$$
w^{\mathrm{FT}}\left(\mathbf{q}_{\|}\right)=\delta\left(\mathbf{q}_{\|}\right)+\sum_{\mathbf{m} \neq(0,0)} P_{|\mathbf{m}| \sigma}\left(\mathbf{q}-m_{1}\left\langle\mathbf{G}_{1}\right\rangle-m_{2}\left\langle\mathbf{G}_{2}\right\rangle\right) .
$$

In reciprocal plane, this distribution creates a square array of Gaussian peaks. With increasing distance from the origin the heights of these peaks decrease and their widths increase. In the origin $\mathbf{q}_{\|}=\mathbf{0}$, this distribution exhibits a sharp $\delta$-like peak.

Let us assume that the relative number $p$ of dots is also correlated vertically and the remainder $1-p$ is vertically not correlated. Then, the scattered intensity equals

$$
\begin{aligned}
I(\mathbf{q})= & w^{\mathrm{FT}}\left(\mathbf{q}_{\|}\right)\left[p\left|\sum_{n=1}^{N} E_{0}\left(\mathbf{q} ; Z_{n}\right) e^{i q_{z} Z_{n}}\right|^{2}\right. \\
& \left.+(1-p) \sum_{n=1}^{N}\left|E_{0}\left(\mathbf{q} ; Z_{n}\right) e^{i q_{z} Z_{n}}\right|^{2}\right] .
\end{aligned}
$$

This formula is used for the intensity simulation.

Equation (11) has been derived using the kinematic scattering theory neglecting absorption and refraction. In the case of GID, the DWBA method ${ }^{23}$ has to be used. Within this method, the scattering centers are immersed in a homogeneous medium (Si matrix in our case). We take absorption and refraction at the sample surface into account but we do 


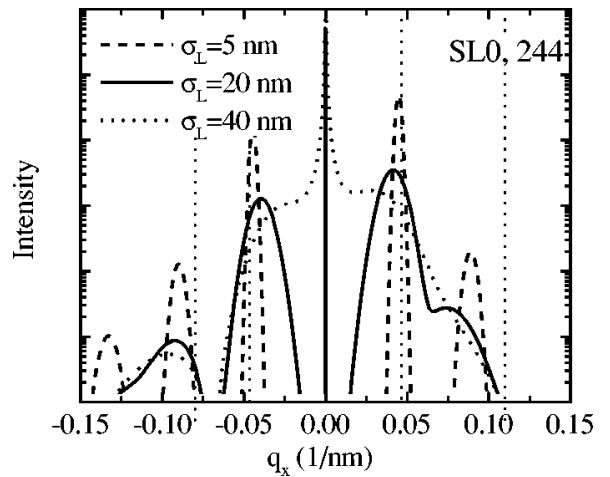

FIG. 13. Intensity distributions along the $q_{x}$ axis calculated for constant $L=100 \mathrm{~nm}$ and various $\sigma_{L}$. The vertical dotted lines show the positions of the lateral satellite maxima for $\sigma_{L}=0$.

not consider the crystalline structure of this medium, i.e., we neglect diffuse scattering of the diffracted wave and/or diffraction of the diffusely scattered wave. Then the scattering vector q occurring in Eq. (11) has to be corrected

$$
\mathbf{q}=\mathbf{k}_{\text {out }}-\mathbf{k}_{\text {in }}-\mathbf{h},
$$

where $\mathbf{k}_{\text {in,out }}$ are the complex wave vectors of the waves inside the Si matrix excited by the incident and scattered vacuum waves, respectively. Additionally, the intensity obtained by Eq. (10) has to be multiplied by $\left|T_{\text {in }} T_{\text {out }}\right|^{2}$, where $T_{\text {in,out }}$ are the transmittivity coefficients of the surface of the Si matrix for the incident and the scattered waves.

\section{DISCUSSION}

Using the above model we have simulated the RSM's of sample SiGe51 in both coplanar and GID geometries [panel (b) in Figs. 3-5 and 8-10]. Some structure parameters occurring in the model can be directly determined from the comparison of the experimental and calculated RSM's. From the position and the shape of the lateral satellites in coplanar and GID RSM's we determined the mean distance $L$ of the dots $L=110 \pm 10 \mathrm{~nm}$ for sample SiGe51 as well as its root mean square deviation $\sigma_{L}=30 \pm 10 \mathrm{~nm}$. $L$ is the mean dot distance along the $\langle 100\rangle$ direction and thus by a factor of $\sqrt{2}$ smaller than that measured along the $\langle 110\rangle$ direction obtained by the cross-sectional TEM; see Sec. II A.

The same values follow from the coplanar RSM's, where the lateral satellites can also be observed. In this geometry, the lateral satellites are more distinct in the region close to the zeroth multilayer satellite. Because of the strain field between the dots (see below) and limited correlation of the dot positions, only the positive first satellite is visible. Its distance $\Delta q_{x}$ form the origin in (404) diffraction appears $\sqrt{2}$ times larger compared to the (113) and (224) RSM's [see Fig. 6(a)]. Moreover, the position of the lateral satellite does not depend on the in-plane component of the diffraction vector. The values of $L$ and $\sigma_{L}$ determined from the coplanar maps coincide perfectly with those following from GID.

The dot distance $L$ cannot be determined only from the position of the lateral satellites since this position is also influenced by its deviation $\sigma_{L} \cdot{ }^{28}$ This effect is demonstrated in Fig. 13, where we have plotted the intensity distribution along the $q_{x}$ axis (for $q_{z}=0$ ), coplanar diffraction (224),

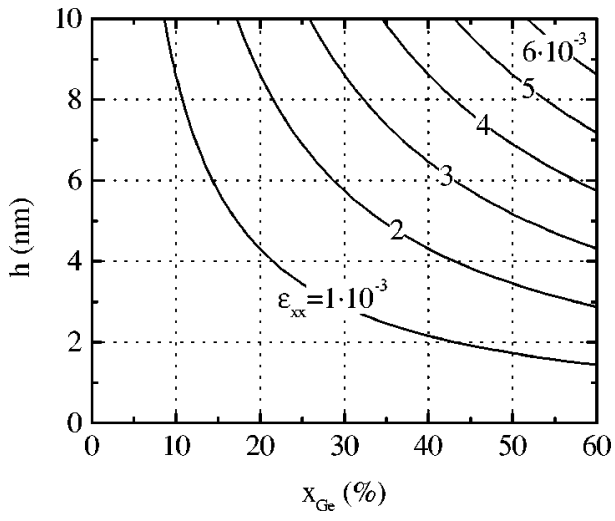

FIG. 14. Dependence of the in-plane strain $\varepsilon_{x x}$ on $h$ and $x_{\mathrm{Ge}}$. The calculation has been performed in the center of a dot lying in the first dot layer under the sample surface.

calculated for various $\sigma_{L}$, and the constant $L=100 \mathrm{~nm}$. For $\sigma_{L} \rightarrow 0$ the position of the $n$th lateral satellite approaches $q_{x n} \rightarrow 2 \pi n / L \sqrt{2}$, whereas for increasing $\sigma_{L}$ a decrease in $q_{x n}$ is observed. Similar behavior can also be found in the GID geometry.

From the measured RSM's the in-plane arrangement of the dots can be reconstructed as follows. The dots are arranged in a square array aligned parallel to the $\langle 100\rangle$ directions. The limited lateral correlation over just 3-4 dots gives sharp lateral satellites of first order along $\langle 100\rangle$. The intensity of the second-order satellite along $\langle 110\rangle$ is several orders of magnitude lower and the third order disappears completely. The values of $L$ of the other samples were determined in the same way and are listed in Table I.

The intensity distribution in the vertical $q_{z}$ direction can be seen from coplanar maps (Figs. 3-5) and from the $q_{y}-q_{z}$ RSM in GID geometry in Fig. 9. The intensity is concentrated into horizontal sheets in the vicinity of the multilayer satellites. The presence of the sheets indicates a strong vertical correlation of the dot positions. This behavior is analogous to the diffuse reflection from rough multilayers, ${ }^{24,29}$ where the correlation of the roughness profiles of different interfaces gives rise to a similar shape of the scattered intensity distribution. From the width of these sheets the percentage $p$ of vertically correlated dots can be estimated; we found $p=0.9 \pm 0.1$, i.e., nearly all the dots are vertically correlated.

The values of other structure parameters in the model, namely, the dot radius $R$, the dot height $h$, and the germanium concentration in the dots $x_{\mathrm{Ge}}$, cannot be determined from the measured data independently since the RSM's are sensitive only to their combinations. In the calculations, we took $R=40 \mathrm{~nm}$ for sample $\mathrm{SiGe} 51$; this value follows from the TEM observation (see Fig. 1). The value of the other parameters can be estimated from the mean in-plane strain $\left\langle\varepsilon_{x x}\right\rangle$ that affects the in-plane RSM's in the GID geometry in Figs. 8 and 10. For sample SiGe51 we have found $\left\langle\varepsilon_{x x}\right\rangle$ $=(1.2 \pm 0.2) \times 10^{-3}$. This value of strain can be achieved using various combinations of $x_{\mathrm{Ge}}$ and $h$; it is illustrated in Fig. 14, where we have plotted the calculated in-plane strain $\varepsilon_{x x}$ as a function of $h$ and $x_{\mathrm{Ge}}$ for constant $R=40 \mathrm{~nm}$. The height of the islands was estimated from the TEM to be about $4 \mathrm{~nm}$. Since in the calculations cylindrical instead of 
lens-shaped islands have been assumed, the height of the cylinders was set to be $h=2 \mathrm{~nm}$. For this value a Ge content of $x_{\mathrm{Ge}}=0.45$ follows according to Fig. 14. Within the uncertainty this agrees reasonable well with the average Ge content obtained from the HRXRD measurements.

Using the above values of the parameters we have simulated both the coplanar and GID RSM's. We achieved a very good qualitative correspondence to the measured data. We did not use the strain components $\varepsilon_{x x, z z}$ in the dots as free parameters, but we rather calculated them from the dot shape and germanium concentration using elasticity theory [Eqs. (1) and (2)]. These strains affect mainly the regions in reciprocal space with larger $|\mathbf{q}|$, therefore, close to the nonzero superlattice satellites. However, due to the vertical correlation of the dot positions the intensity in this region is concentrated in horizontal sheets. This fact makes it impossible to determine a tiny vertical shift of the intensity maximum due to $\varepsilon_{z z}$.

The intensity distribution in the sheet crossing the first satellite is shifted to negative $q_{x}$ values. It corresponds therefore to the lateral dilatation of the lattice in the dot volumes. If the intensity distribution depended on $\varepsilon_{x x}$ only, the position of its maximum would be proportional to the in-plane component $\left|\mathbf{h}_{\|}\right|$of the diffraction vector. It is obvious from Fig. 6(b) that this position only slightly depends on $\left|\mathbf{h}_{\|}\right|$; therefore, the intensity maximum is rather influenced by the lateral intensity satellites due to the dot arrangement. Due to this fact, a direct determination of $\varepsilon_{x x}$ from a coplanar map is not possible. However, the numerical simulations take all the effects into account and the experimental intensity distributions in Fig. 6(b) coincide very well with the calculations performed for the same parameter values.

The intensity distributions around the zeroth-order satellites in asymmetrical diffractions are shifted in the opposite direction, i.e., towards positive $q_{x}$. This shift is caused by the compressive strain between the dots. In the simulated RSM's similar asymmetry occurs; however, the measured intensity distributions in the $q_{x}$ direction do not agree completely with the simulations. The discrepancy might be caused by assuming a cylindrical shape of the dots instead of the real one, so that the calculated deformation field around it differs from the actual one.

In-plane ordering of the dot positions gives rise to lateral intensity satellites around any multilayer satellite. Their positions are not affected by the deformation field inside and/or outside the dots. Similar effects have been found for the case of etched dot structures. ${ }^{29}$ It has been demonstrated that the positions of the lateral satellites are insensitive to the strains if the structure remains pseudomorphic. Thus, if the twodimensional dot array were perfectly ordered, the lateral satellites would be very narrow and the strains in the structure would cause only a shift of the envelope curve of these satellites. From Fig. 7 we obtained the trend of the mean dot distance with Ge content $x_{\mathrm{Ge}}$ and growth temperature consistent with earlier observations. ${ }^{11}$ From the intensity distribution close to the zeroth satellite [Fig. 7(a)] it follows that the dot separation $L$ increases with growth temperature as the dots become larger. The dependence of $L$ on Ge concentration $x_{\mathrm{Ge}}$ is not obvious from our data. Samples SiGe53 and SiGe51 differ in the Ge content and they have been grown at the same temperature. Their $L$ values coincide within the

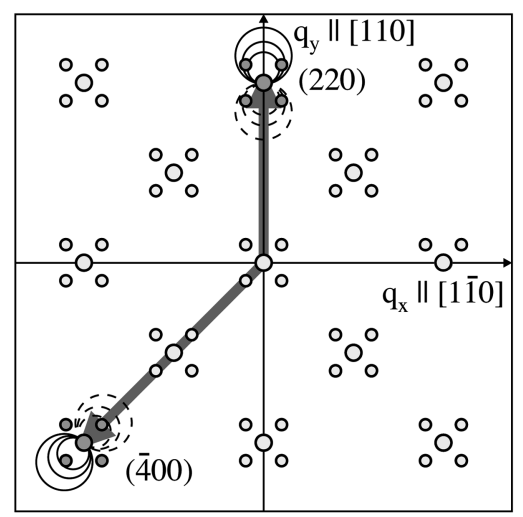

FIG. 15. Schematic diagram to explain the observed intensity distribution in Figs. 8 and 10. The dots represent the correlation function of the lateral ordered island structure. The lobes correspond to the scattering factor, determined by the product of the scattering vector $\mathbf{h}$ and the deviation $\mathbf{q}$ from the Bragg condition $(\mathbf{h} \cdot \mathbf{q})$. The resulting intensity distribution is a convolution of these two terms, thus in the (400) RSM the satellite maxima along [010] are suppressed. For the (220) RSM, all four subsidiary maxima are visible.

error limits; however, the difference of $10 \%$ in $x_{\mathrm{Ge}}$ is not large enough to draw any significant conclusion. The interpretation of the distributions around the first multilayer satellite [Fig. 7(b)] is not straightforward since it is influenced by both the dot distance $L$ and the strain $\varepsilon_{x x}$ inside the dots. The trend of the positions of the lateral maxima is similar to that in Fig. 7(a).

This lateral ordering is also observed in GID reciprocal space maps in the $q_{x}-q_{y}$ plane. The different shape of the (220) and (400) GID maps in Figs. 8 and 10 may be explained by the nature of the scattering factor. Because the penetration depth of the incoming radiation is smaller than the thickness of the upper capping layer, the scattering contrast is caused mainly by the strain propagating from the topmost dot layer towards the surface. This strain field reflects the periodicity of the dot positions that gives rise to lateral satellites. The lateral satellites are clearly visible in Fig. 8; from their distance the same mean dot distance $L$ $=110 \mathrm{~nm}$ follows as in coplanar HRXRD. Only four lowestorder lateral satellites can be seen; the higher satellites are suppressed.

Apart from the lateral satellites, the intensity distribution in the $q_{x}-q_{y}$ plane is determined by the strain field around a single dot. From Eq. (4) it follows that, close to the origin of reciprocal space, it is mainly given by the factor

$$
\frac{|\mathbf{h} \cdot \mathbf{q}|^{2}}{|\mathbf{q}|^{4}}
$$

Therefore, a nodal plane of the scattered intensity exists for $\mathbf{h} \perp \mathbf{q}$ for which $\mathbf{h} \cdot \mathbf{q}=0$; the intensity distribution is analogous to the Huang scattering from point defects. ${ }^{27,30}$ In (220) diffraction, all the four visible lateral satellites lie outside this plane. In (400) diffraction, however, two of the lateral satellites lie in the nodal plane and therefore they are suppressed (Fig. 10). In this diffraction, the nodal plane of the intensity can also be seen. The situation is sketched schematically in Fig. 15. 
For larger incidence angles $\alpha_{i}$ the penetration depth of the primary radiation is larger than the thickness $T_{c}$ of the capping layer. Then the scattered intensity is also sensitive to the contrast of the polarizability $\chi_{h}(\mathbf{r})$. This contribution to the scattered intensity is analogous to the Stokes-Wilson scattering from the defects cores ${ }^{27}$ and it exhibits no nodal plane. In this case, all four lateral satellites would be visible even in ( $\overline{4} 00)$ diffraction.

Figure 9 shows the measured $q_{y}-q_{z}$ RSM in (220) GID diffraction. Similarly to the coplanar HRXRD, the diffusely scattered intensity is concentrated in the sheets crossing the superlattice satellites. This indicates a nearly perfect vertical correlation of the dot positions. Along a particular sheet, the intensity maximum is shifted to a negative $q_{y}$ value. From the shift, the mean in-plane strain $\left\langle\varepsilon_{x x}\right\rangle$ in the dot volumes can be deduced; its value $1.2 \times 10^{-3}$ is the same as that following from GID in-plane RSM's. Increasing $q_{z}$ in this RSM we increase also the penetration depth of the primary radiation. Since no distinct differences in the positions of the intensity maxima in different superlattice satellites can be observed, the in-plane strains in different dot layers do not differ substantially. This finding agrees with Fig. 12, where the calculated strains $\varepsilon_{x x, z z}$ are plotted.

\section{CONCLUSION}

A theory has been derived that describes the diffuse x-ray scattering from self-organized dots both in coplanar and in grazing incidence high-resolution $\mathrm{x}$-ray diffraction. With one set of parameters we could consistently describe the intensity distribution in both scattering geometries. The simulated intensity distributions agree indeed very well with the measured GID and HRXRD reciprocal space maps. The calculated inhomogeneous strain fields in the Ge-rich islands in $\mathrm{Si} / \mathrm{SiGe}$ multilayers correspond closely to those determined directly from GID. Reciprocal space maps measured in coplanar geometry show that the diffusely scattered intensity is concentrated in the vicinity of the multilayer satellites due to the high degree of vertical correlation. The strains in the island volumes mainly affect the regions in reciprocal space around the higher-order superlattice satellites. The shift of the intensity distribution towards smaller values of $q_{x}$ observed in the reciprocal space maps around the (113), (224), and (404) reciprocal lattice points corresponds to the lateral lattice dilation in the Ge islands $\varepsilon_{x x}$. The intensity distribution around the zeroth-order satellite is mainly caused by the compressive strain in the Si matrix between the islands and by the dilatative strain both below and above the islands.
With grazing incidence diffraction, which was measured for the (220) and (400) reciprocal lattice points, the in-plane relaxation, the lateral ordering, and information on the vertical correlation were obtained.

This demonstrates that high-resolution x-ray-diffraction techniques (HRXRD and GID) are very suitable for the investigation of the morphology and strains in self-organized dot structures. Using GID we can visualize the reciprocal lattice of the two-dimensional array of dots. Thus the statistical parameters of the dot distribution such as the mean dot distance and the crystallographic orientation as well as their root mean square deviations can be determined for a comparatively large sample area. Such information is required for any possible application. The GID technique is also sensitive to the mean in-plane strain in the dot volumes. With coplanar HRXRD the intensity distribution close to the reciprocal lattice point of the averaged structure can be recorded, which is not possible with GID due to refraction. Thus, using HRXRD we are able to study also the deformation field in the Si matrix around the dots.

The limitation of both diffraction methods is their relatively weak sensitivity to the shape of the buried dots and to their chemical composition. This information can be deduced only indirectly from the experimentally obtained strains; however, this cannot yield an unambiguous result. In order to study the shape of the dots and their chemical composition, other methods must be used. Attempts to obtain locally resolved information on the chemical composition with scanning transmission electron microscopy using energy dispersive $\mathrm{x}$-ray analysis are in progress. ${ }^{31}$ Recently, it was shown that with convergent beam electron measurements, ${ }^{32}$ the displacement fields inside and around Ge islands on Si are accessible. Furthermore, attempts have been reported to reveal the depth-dependent chemical composition of islands in $\mathrm{CdSe} / \mathrm{ZnSe}$ structures by analyzing digitized high-resolution TEM images. ${ }^{33}$ It seems that only a combination of several techniques will yield a complete and consistent picture of the strain fields, shape, and compositions of the islands.

\section{ACKNOWLEDGMENTS}

One of us (V.H.) appreciates the financial support of the Grant Agency of the Czech Republic (Project No. 202/97/ 0003) and the Ministry of Education of the Czech Republic (Project No. VS96102). This work was supported by FWF (Projects Nos. 10083 and 11557PHY), GMe, and BMWV, Vienna. We are grateful to T. H. Metzger for discussions on GID measurements.
*Permanent address: Laboratory of Thin Films and Nanostructures, Masaryk University, Kotlárská 2, 61137 Brno, Czech Republic.

†Permanent address: Fraunhofer Institut für Zerstörungsfreie Prüfverfahren, D-01326 Dresden, Germany.

${ }^{1}$ W. Seifert, N. Carlsson, M. Miller, M.-E. Pistol, L. Samuelson, and L. R. Wallenberg, Prog. Cryst. Growth Charact. Mater. 33, 423 (1996), and references therein.

${ }^{2}$ D. E. Jesson, K. M. Chen, S. J. Pennycook, T. Thundat, and R. J. Warmack, Phys. Rev. Lett. 77, 1330 (1996).

${ }^{3}$ For a recent review see, e.g., F. Schäffler, Semicond. Sci. Technol. 12, 1515 (1997), and references therein.
${ }^{4}$ C. Teichert, Y. H. Phang, L. J. Peticolas, J. C. Bean, and M. G. Lagally, in Surface Diffraction: Atomistic and Collective Processes, edited by M. C. Tringides (Plenum, New York, 1997), p. 297.

${ }^{5}$ C. Teichert, J. Tersoff, and M. G. Lagally, in Morphological Organization in Epitaxial Growth and Removal, edited by Z. Zhang and M. G. Lagally (World Scientific, Singapore, in press).

${ }^{6}$ K. H. Schmidt, G. Medeiros-Ribeiro, M. Oestreich, P. M. Petroff, and G. H. Döhler, Phys. Rev. B 54, 11346 (1996), and references therein. 
${ }^{7}$ Q. Xie, A. Madhukar, P. Chen, and N. P. Kobayashi, Phys. Rev. Lett. 75, 2542 (1995).

${ }^{8}$ A. A. Darhuber, V. Holý, J. Stangl, G. Bauer, A. Krost, F. Heinrichsdorff, M. Grundmann, D. Bimberg, V. M. Ustinov, P. S. Kop'ev, A. O. Kosogov, and P. Werner, Appl. Phys. Lett. 70, 955 (1997).

${ }^{9}$ J. Tersoff, C. Teichert, and M. G. Lagally, Phys. Rev. Lett. 76, 1675 (1996).

${ }^{10}$ C. Teichert, M. G. Lagally, L. J. Peticolas, J. C. Bean, and J. Tersoff, Phys. Rev. B 53, 16334 (1996).

${ }^{11}$ P. Schittenhelm, G. Abstreiter, A. A. Darhuber, G. Bauer, P. Werner, and A. Kosogov, Thin Solid Films 294, 291 (1997).

${ }^{12}$ F. Liu, J. Tersoff, and M. G. Lagally, Phys. Rev. Lett. 80, 1268 (1998)

${ }^{13}$ J. Tersoff, Phys. Rev. Lett. 77, 2017 (1996).

${ }^{14}$ A. A. Darhuber, P. Schittenhelm, V. Holý, J. Stangl, G. Bauer, and G. Abstreiter, Phys. Rev. B 55, 15652 (1997).

${ }^{15}$ J.-M. Baribeau, J. Cryst. Growth 157, 52 (1995).

${ }^{16}$ U. Pietsch, T. H. Metzger, S. Rugel, B. Jenichen, and I. K. Robinson, J. Appl. Phys. 74, 2381 (1993).

${ }^{17}$ A. A. Williams, J. M. C. Thornton, J. E. Macdonald, R. G. van Silfhout, J. F. van der Veen, M. S. Finney, A. D. Johnson, and C. Norris, Phys. Rev. B 43, 5001 (1991).

${ }^{18}$ A. J. Steinfort, P. M. L. O. Scholte, A. Ettema, F. Tuinstra, M. Nielsen, E. Landemark, D.-M. Smilgies, R. Feidenhans'1, G. Falkenberg, L. Seehofer, and R. L. Johnson, Phys. Rev. Lett. 77, 2009 (1996).

${ }^{19}$ A. A. Darhuber, V. Holý, P. Schittenhelm, J. Stangl, I. Kegel, Z.
Kovats, T. H. Metzger, G. Bauer, G. Abstreiter, and G. Grübel, Physica B (to be published).

${ }^{20}$ G. Medeiros-Ribeiro, A. M. Bratkovski, T. I. Kamins, D. A. A. Ohlberg, and R. Stanley Williams, Science 279, 353 (1998).

${ }^{21}$ I. Kegel, T. H. Metzger, P. Fratzl, J. Peisl, A. Lorke, J. P. Kotthaus, and P. M. Petroff (unpublished).

${ }^{22}$ See, e.g., Q. Shen, C. C. Umbach, B. Weselak, and J. M. Blakely, Phys. Rev. B 53, R4237 (1996).

${ }^{23}$ S. K. Sinha, E. B. Sirota, S. Garoff, and H. B. Stanley, Phys. Rev. B 38, 2297 (1988).

${ }^{24}$ V. Holý and T. Baumbach, Phys. Rev. B 49, 10668 (1994).

${ }^{25}$ V. Holý, U. Pietsch, and T. Baumbach, X-Ray Scattering by Thin Films and Multilayers (Springer-Verlag, Berlin, in press).

${ }^{26}$ S. M. Hu, J. Appl. Phys. 66, 2741 (1989).

${ }^{27}$ M. A. Krivoglaz, Diffraction of X-Rays and Neutron in Non-Ideal Crystals (Springer-Verlag, Berlin, 1996).

${ }^{28}$ P. R. Pukite, C. S. Lent, and P. I. Cohen, Surf. Sci. 161, 39 (1985).

${ }^{29}$ V. Holý, A. A. Darhuber, G. Bauer, P. D. Wang, Y. S. Song, C. M. Sotomayor Torres, and M. C. Holland, Phys. Rev. B 52, 8348 (1995).

${ }^{30}$ K. Huang, Proc. R. Soc. London, Ser. A 190, 102 (1947).

${ }^{31}$ O. Kienzle (private communication).

${ }^{32}$ S. Christiansen, M. Albrecht, H. P. Strunk, and H. J. Maier, Appl. Phys. Lett. 64, 3617 (1994).

${ }^{33}$ M. Strassburg, V. Kutzer, U. W. Pohl, A. Hoffmann, I. Broser, N. N. Ledentsov, D. Bimberg, A. Rosenauer, U. Fischer, D. Gerthsen, I. L. Krestnikov, M. V. Maximov, P. S. Kop'ev, and Zh. I. Alferov, Appl. Phys. Lett. 72, 942 (1998). 